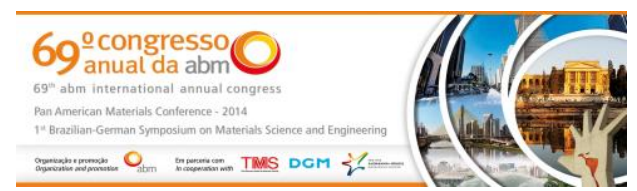

Tema: Gestão Sistêmica

\title{
ANÁLISE DA CONFIABILIDADE NA DETERMINAÇÃO DAS PROPRIEDADES MECÂNICAS EM ENSAIOS DE TRAÇÃO*
}

\author{
Cláudia Lisiane Fanezi da Rocha ${ }^{1}$ \\ Daniel Antonio Kapper Fabricio ${ }^{2}$ \\ Jefferson $\mathrm{Haag}^{3}$ \\ Telmo Roberto Strohacker ${ }^{4}$
}

\begin{abstract}
Resumo
Softwares desenvolvidos para análise das propriedades mecânicas em máquinas de ensaio de tração facilitam o trabalho rotineiro de caracterização dos materiais. No entanto, estes aplicativos não apresentam informações importantes como cálculos da incerteza de medição, cabendo uma análise da confiabilidade dos resultados obtidos por esses programas. O presente trabalho se propõe a comparar os valores dos limites de escoamento, de resistência, do módulo de elasticidade e da deformação final obtidos através do software Instron Bluehil ${ }^{\circledR}$ com os valores calculados através da análise minuciosa dos dados provenientes dos instrumentos de medição (célula de carga, extensômetro, sensor de deslocamento). Busca-se, assim, fornecer informações que auxiliem o trabalho de técnicos e engenheiros, além de estimar o erro percentual e desenvolver uma metodologia periódica para a garantia da confiabilidade da máquina de ensaio.
\end{abstract}

Palavras-chave: Propriedades Mecânicas; Ensaio de Tração; Confiabilidade.

\section{RELIABILITY ANALYSIS IN THE DETERMINATION OF MECHANICAL PROPERTIES IN TESTILE TESTS}

\begin{abstract}
There are computational programs developed for the analysis of mechanical properties in tensile testing machines, which facilitate the routine to determine material properties. However, these programs do not present important data such as the estimation of their measurement uncertainty, requiring an analysis of the reliability of the results obtained. This work aims to compare the values of yield strength, tensile strength, modulus of elasticity and total extension obtained from Instron Bluehill ${ }^{\circledR}$ software with the values calculated by the direct analysis of data from the measuring instruments (load cell, extensometer and displacement sensor). Thus, this paper intends to provide information to help technicians and engineers, and also to estimate the relative error and develop a periodical methodology to ensure the reliability of the testing machine.
\end{abstract}

Keywords: Mechanical Properties; Tensile Test; Reliability.

\footnotetext{
Engenheira Metalúrgica, Doutoranda, PPGE3M, UFRGS, Porto Alegre, RS, Brasil. Engenheiro de Produção, Mestrando, PPGE3M, UFRGS, Porto Alegre, RS, Brasil Engenheiro Metalúrgico, Mestrando, PPGE3M, UFRGS, Porto Alegre, RS, Brasil.

Engenheiro Metalúrgico, Professor Doutor, PPGE3M, UFRGS, Porto Alegre, RS, Brasil.
}

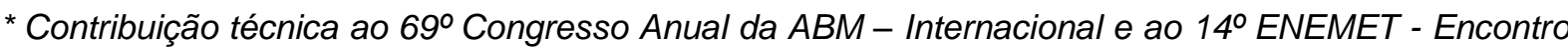
Nacional de Estudantes de Engenharia Metalúrgica, de Materiais e de Minas, 21 a 25 de julho de 2014, São Paulo, SP, Brasil.
} 


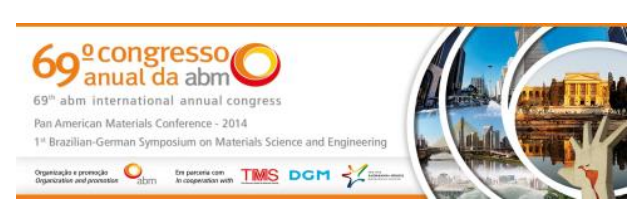

\section{INTRODUÇÃO}

O ensaio de tração é amplamente utilizado na indústria de componentes mecânicos, devido à vantagem de fornecer dados de uma ampla gama de propriedades mecânicas dos materiais. Além disso, o ensaio é relativamente simples e de realização rápida, podendo ser aplicado a diversos tipos de materiais de engenharia. $\mathrm{Na}$ execução do ensaio, a curva tensão-deformação convencional é obtida por carregamento uniaxial estático em corpos de prova padronizados [1].

Os ensaios de tração são rotineiramente aplicados na caracterização dos diversos tipos de materiais existentes. Os resultados oriundos dos ensaios são influenciados por diversas variáveis como: temperatura, taxa de deformação, anisotropia do material, tamanho de grão, porcentagem de impurezas e condições ambientais [2-4]. Ao executar o ensaio em máquinas universais, há programas computacionais que facilitam a aquisição e análise dos dados do ensaio. Em particular, o software Instron Bluehil/ ${ }^{\circledR}$, utilizado nesse trabalho, determina automaticamente algumas propriedades do ensaio de tração, tais como os limites de escoamento e resistência. Para verificar a exatidão dos valores das propriedades calculadas pelo software, é de fundamental importância comparar os resultados obtidos com outro método de referência. A determinação dessas propriedades pode ser realizada por meio de planilhas eletrônicas, tratando-se os dados brutos adquiridos durante o ensaio.

Para análise dos dados, pode-se utilizar simplesmente o erro percentual entre os valores medidos. Para uma análise mais completa, é importante utilizar métodos estatísticos, tais como a Análise de Variância (Analysis of Variance - ANOVA). A ANOVA é uma metodologia que avalia a significância dos diversos fatores e interações. Em particular, a ANOVA de dois fatores (two-way ANOVA) busca identificar se os valores da média da variável de resposta diferem entre si devido ao efeito de dois fatores controláveis [5].

Portanto, o presente trabalho se propõe a comparar os valores dos limites de escoamento e de resistência obtidos através do software Instron Bluehil ${ }^{\circledR}$ com os valores obtidos diretamente dos instrumentos de medição, através do tratamento desses dados. Busca-se, assim, validar ou não os resultados calculados automaticamente pelo software.

\section{MATERIAIS E MÉTODOS}

Foram realizados ensaios de tração conforme previsto na norma ASTM E8/E8M [6], através de uma máquina eletromecânica marca Instron modelo $5585 \mathrm{H}$ com capacidade para $250 \mathrm{kN}$ (25 toneladas). Tais ensaios foram executados a temperatura ambiente (aproximadamente $23^{\circ} \mathrm{C}$ ).

Foram ensaiados corpos de prova (CPs) de seção retangular (Figura 1) e corpos de prova de seção circular (Figura 2). Para cada geometria, foram ensaiadas três amostras com escoamento contínuo e três com escoamento descontínuo.

As amostras estão identificadas com a seguinte estrutura: $X-Y-Z$, onde:

- X representa a geometria do corpo de prova, sendo C para Circular e R para Retangular;

- Y representa o patamar de escoamento, onde $\mathrm{C}$ indica um escoamento Contínuo e D um escoamento Descontínuo;

- Z representa o número do corpo de prova dentro de uma mesma condição $(1,2,3)$.

\footnotetext{
* Contribuição técnica ao $69^{\circ}$ Congresso Anual da ABM - Internacional e ao 14ํㅡㄹ ENEMET - Encontro Nacional de Estudantes de Engenharia Metalúrgica, de Materiais e de Minas, 21 a 25 de julho de 2014, São Paulo, SP, Brasil.
} 

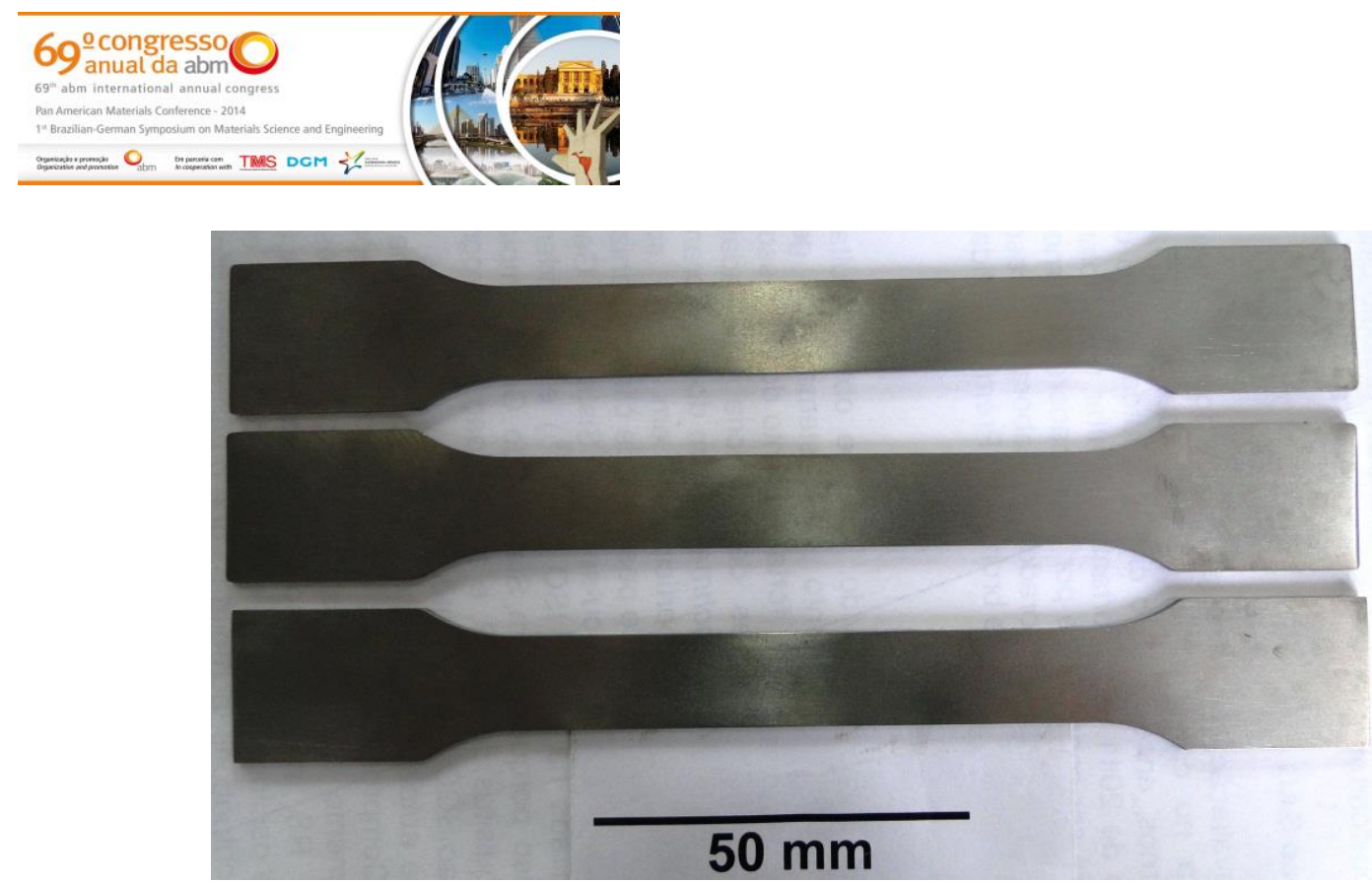

Figura 1. Corpos de prova de seção retangular.

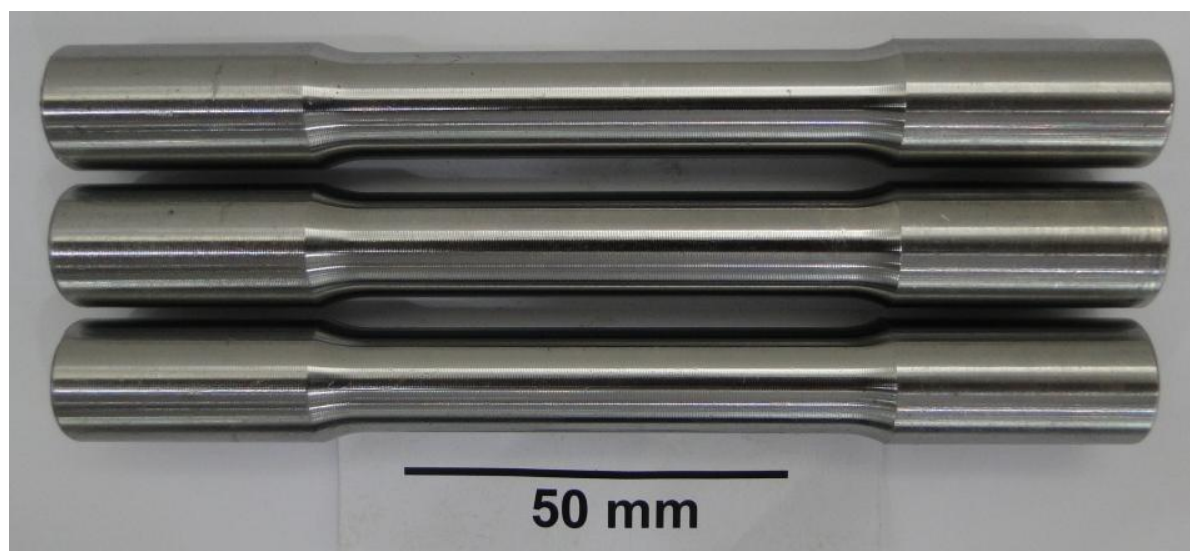

Figura 2. Corpos de prova de seção circular.

A Tabela 1 apresenta a identificação adotada para os doze corpos de prova ensaiados.

Tabela 1. Identificação dos corpos de prova

\begin{tabular}{ccc}
\hline Identificação do CP & Geometria da Seção & Tipo de Escoamento \\
\hline C-C-1 & Circular & Contínuo \\
\hline C-C-2 & Circular & Contínuo \\
\hline C-C-3 & Circular & Contínuo \\
\hline R-C-1 & Retangular & Contínuo \\
\hline R-C-2 & Retangular & Contínuo \\
\hline R-C-3 & Retangular & Contínuo \\
\hline C-D-1 & Circular & Descontínuo \\
\hline C-D-2 & Circular & Descontínuo \\
\hline C-D-3 & Circular & Descontínuo \\
\hline R-D-1 & Retangular & Descontínuo \\
\hline R-D-2 & Retangular & Descontínuo \\
\hline R-D-3 & Retangular & Descontínuo
\end{tabular}

Os dados do ensaio foram tratados e analisados através de planilhas eletrônicas (método I), e em seguida foram comparados com os valores obtidos automaticamente através do software Instron Bluehil ${ }^{\circledR}$ (método II).

\footnotetext{
* Contribuição técnica ao $69^{\circ}$ Congresso Anual da ABM - Internacional e ao 14ํㅡㄹ ENEMET - Encontro Nacional de Estudantes de Engenharia Metalúrgica, de Materiais e de Minas, 21 a 25 de julho de 2014, São Paulo, SP, Brasil.
} 


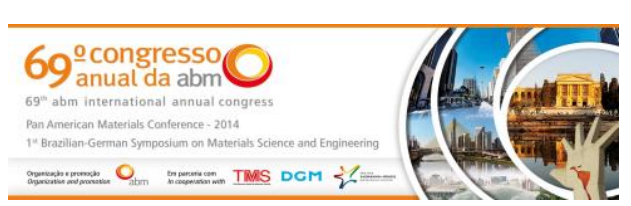

O procedimento utilizado para o tratamento de dados em planilhas eletrônicas é apresentado em uma representação esquemática na Figura 3 e na Figura 4. Esse procedimento é baseado nas normas ASTM E8/E8M [6] e ISO 6892-1 [7]. Para as amostras com escoamento contínuo, o limite de escoamento $\left(\sigma_{e}\right)$ é calculado traçando uma reta paralela à região linear, utilizando o método offset e o limite $n$, este que é, normalmente $0,2 \%$ para metais e ligas. A tensão referente à intersecção entre a reta paralela e a curva tensão-deformação é o limite de escoamento do material. As amostras que apresentaram escoamento descontínuo tiveram o limite de escoamento calculado diretamente através da tensão mínima alcançada no patamar de escoamento. O limite de resistência $\left(\sigma_{u}\right)$ é o valor máximo da tensão alcançada no ensaio de tração. O módulo de elasticidade $(E)$ é calculado através da regressão dos pontos na região linear da curva tensão-deformação. Por fim, a deformação final $\left(\varepsilon_{f}\right)$ é o último ponto de deformação da curva antes da fratura ocorrer, ou seja, a queda abrupta da tensão a subsequente deformação do corpo de prova.

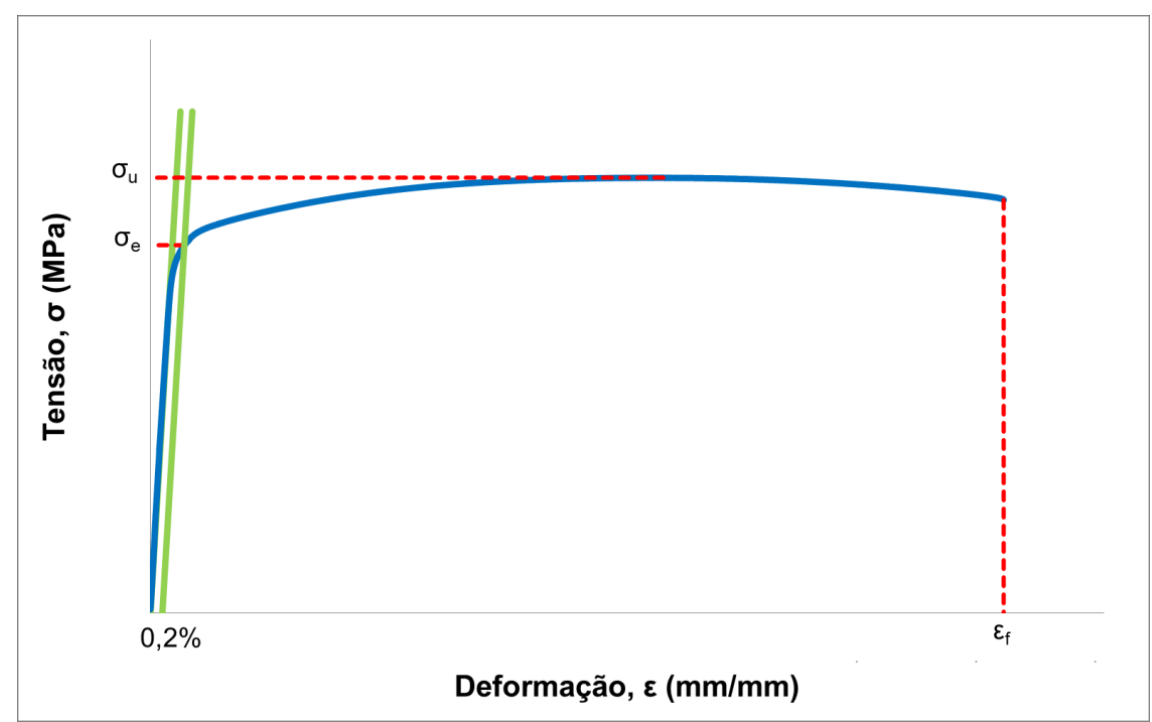

Figura 3. Representação esquemática do tratamento de dados por planilhas eletrônicas com escoamento contínuo.

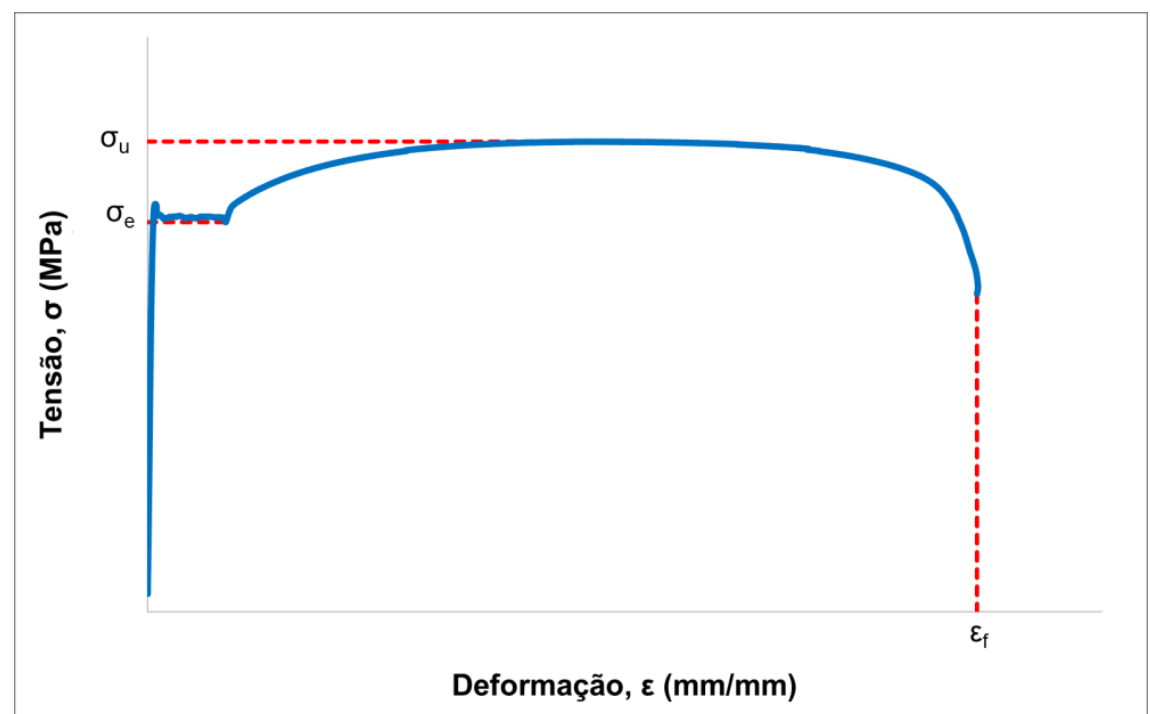

Figura 4. Representação esquemática do tratamento de dados para amostra com escoamento descontínuo.

* Contribuição técnica ao $69^{\circ}$ Congresso Anual da ABM - Internacional e ao 14ํㅡㄹ ENEMET - Encontro Nacional de Estudantes de Engenharia Metalúrgica, de Materiais e de Minas, 21 a 25 de julho de 2014, São Paulo, SP, Brasil. 


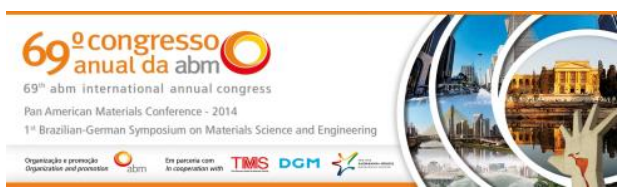

Para cada uma das doze amostras ensaiadas, foram determinados os valores das propriedades $\sigma_{e}, \sigma_{u}, E$ e $\varepsilon_{f}$. Cada uma dessas propriedades foi obtida pelo método direto (resultado calculado automaticamente pelo equipamento) e por filtragem (tratamento) dos dados, sendo comparados os seus respectivos valores.

Para a comparação entre os resultados tratados e os valores obtidos diretamente da máquina, foi realizado o cálculo do erro percentual $(E P \%)$ entre os mesmos, assumindo o resultado calculado em planilha eletrônica como valor referência $(V R)$ e o resultado obtido pela máquina como valor aproximado (VA). Esse cálculo é demonstrado na Equação 1.

$$
E P \%=\frac{|V R-V A|}{V R} * 100 \%
$$

Complementarmente, foi realizada a two-way ANOVA entre os valores obtidos, visando verificar a significância das eventuais diferenças obtidas entre os métodos. Foram considerados os seguintes fatores controláveis:

- Método de cálculo: análise via planilha eletrônica (método I) e valor da máquina (método II);

- Tipo de ensaio: corpo de prova circular com escoamento contínuo, corpo de prova retangular com escoamento contínuo, CP circular com escoamento descontínuo e CP retangular com escoamento descontínuo,

A ANOVA foi realizada com nível de significância de $95 \%$ e, como critério de aceitação, foi analisado se os valores de $F$ calculados foram inferiores aos valores estatísticos $F$ tabelados.

\section{RESULTADOS E DISCUSSÃO}

Os resultados do ensaio de tração dos doze corpos de prova se encontram na Tabela 2. Na tabela, as colunas identificadas com I representam os valores tratados, e as colunas com II são os valores obtidos diretamente da máquina de ensaio.

Tabela 2. Resultados dos ensaios

\begin{tabular}{ccccccccc}
\hline \multirow{2}{*}{$\mathrm{CP}$} & \multicolumn{2}{c}{$\sigma_{e}[\mathrm{MPa}]$} & \multicolumn{2}{c}{$\sigma_{u}[\mathrm{MPa}]$} & \multicolumn{2}{c}{$E[\mathrm{MPa}]$} & \multicolumn{2}{c}{$\varepsilon_{f}[\%]$} \\
\cline { 2 - 9 } & $\mathrm{I}$ & $\mathrm{II}$ & $\mathrm{I}$ & $\mathrm{II}$ & $\mathrm{I}$ & $\mathrm{II}$ & $\mathrm{I}$ & $\mathrm{II}$ \\
\hline $\mathrm{C}-\mathrm{C}-1$ & 677,69 & 682,26 & 763,98 & 763,98 & 98333,30 & 94139,03 & $16,69 \%$ & $16,16 \%$ \\
\hline $\mathrm{C}-\mathrm{C}-2$ & 681,06 & 684,19 & 765,37 & 765,37 & 94152,81 & 92224,07 & $13,35 \%$ & $14,98 \%$ \\
\hline $\mathrm{C}-\mathrm{C}-3$ & 680,40 & 678,25 & 761,76 & 761,76 & 98288,52 & 127562,48 & $12,98 \%$ & $9,99 \%$ \\
\hline $\mathrm{R}-\mathrm{C}-1$ & 215,75 & 217,02 & 289,92 & 289,92 & 152181,91 & 125636,40 & $27,62 \%$ & $27,50 \%$ \\
\hline R-C-2 & 217,88 & 218,52 & 291,25 & 291,25 & 148684,33 & 131981,58 & $25,21 \%$ & $25,08 \%$ \\
\hline R-C-3 & 224,06 & 224,56 & 295,80 & 295,80 & 148269,53 & 138462,57 & $22,30 \%$ & $21,72 \%$ \\
\hline C-D-1 & 388,73 & 394,92 & 579,19 & 579,19 & 218402,03 & 221318,43 & $25,25 \%$ & $25,23 \%$ \\
\hline C-D-2 & 387,42 & 400,39 & 585,45 & 585,45 & 225779,01 & 227097,58 & $32,96 \%$ & $29,89 \%$ \\
\hline C-D-3 & 395,49 & 409,22 & 589,67 & 589,67 & 202235,00 & 217172,97 & $40,33 \%$ & $25,28 \%$ \\
\hline R-D-1 & 260,90 & 264,55 & 319,39 & 319,39 & 109821,63 & 113081,26 & $33,87 \%$ & $33,24 \%$ \\
\hline R-D-2 & 270,24 & 272,37 & 327,84 & 327,84 & 124060,92 & 128962,17 & $30,37 \%$ & $29,39 \%$ \\
\hline R-D-3 & 219,95 & 219,54 & 348,27 & 348,27 & 82037,57 & 91859,56 & $56,37 \%$ & $53,37 \%$ \\
\hline
\end{tabular}

A Figura 5 apresenta os valores dos erros percentuais encontrados para as propriedades entre os dois métodos de análise.

\footnotetext{
* Contribuição técnica ao $69^{\circ}$ Congresso Anual da ABM - Internacional e ao 14ํㅡㄹ ENEMET - Encontro Nacional de Estudantes de Engenharia Metalúrgica, de Materiais e de Minas, 21 a 25 de julho de 2014, São Paulo, SP, Brasil.
} 
Tabela 5. ANOVA para a deformação final

\begin{tabular}{lcccccc}
\hline Fonte da variação & SQ & gl & MQ & F & valor-P & F crítico \\
\hline Tipo de ensaio & 0,201215 & 3 & 0,067072 & 11,68035 & 0,000264 & 3,238872 \\
\hline Método de cálculo & 0,002702 & 1 & 0,002702 & 0,470618 & 0,502524 & 4,493998 \\
\hline Interações & 0,003208 & 3 & 0,001069 & 0,186198 & 0,904228 & 3,238872 \\
\hline Resíduo & 0,091876 & 16 & 0,005742 & & & \\
\hline Total & 0,299001 & 23 & & & & \\
\hline
\end{tabular}

A Tabela 6 apresenta as conclusões a respeito das três análises de variância realizadas, mostrando quais fatores foram significativos a um nível de confiança de $95 \%$.

Tabela 6. Conclusão das análises

\begin{tabular}{ccc}
\hline Propriedade & Fonte da variação & Influência \\
\hline \multirow{2}{*}{$\sigma_{e}$} & Tipo de ensaio & Significativa \\
\cline { 2 - 3 } & Método de cálculo & Não significativa \\
\cline { 2 - 3 }$E$ & Interação entre os fatores & Não significativa \\
\cline { 2 - 3 } & Tipo de ensaio & Significativa \\
\cline { 2 - 3 } & Método de cálculo & Não significativa \\
\cline { 2 - 3 }$\varepsilon_{f}$ & Interação entre os fatores & Não significativa \\
\cline { 2 - 3 } & Tipo de ensaio & Significativa \\
\cline { 2 - 3 } & Método de cálculo & Não significativa \\
\hline
\end{tabular}

Nos dois casos, os valores estatísticos $F$ calculados foram menores que os valores $F$ críticos para o método de cálculo, sendo assim possível afirmar, a um nível de confiança de $95 \%$, que a diferença entre os métodos de determinação das propriedades não foi superior ao erro aleatório.

Pode-se notar uma diferença significativa entre os tipos de ensaio realizados. Esse resultado era esperado, visto que foram ensaiados materiais diferentes e com corpos de prova de geometrias diferentes.

Apesar de não apresentar diferença significativa entre o método de cálculo pela ANOVA, devem-se avaliar cuidadosamente os fatores que a afetam cada propriedade estudada. Para o limite de escoamento, o erro é esperado deveria ser baixo, visto que o software aplica o método offset com o limite $n$ de $0,2 \%$. Entretanto, como o software não avalia criticamente o patamar de escoamento, é esperado que o erro fosse maior para os materiais que apresentem esse tipo de comportamento. $O$ limite de resistência não mostrou diferenças, o que era esperado em virtude da aquisição da carga máxima ser simples de ser executada. Para o módulo de elasticidade e para a deformação na fratura, o erro é muito maior e isso ocorre por diversos fatores operacionais, como escorregamento do extensômetro durante o ensaio em virtude da estricção e do encruamento do material. Cabe ressaltar que ambos esses parâmetros são apenas estimados pela técnica do ensaio de tração, principalmente, o módulo de elasticidade que precisa de maior precisão na aquisição das deformações se calculado a partir desse método (por exemplo, strain gages colados na superfície da amostra). Dessa forma, responsável pelo ensaio deve observar se desvios operacionais não vão influenciar no resultado final do ensaio de tração.

\footnotetext{
* Contribuição técnica ao $69^{\circ}$ Congresso Anual da ABM - Internacional e ao 14ํㅡㄹ ENEMET - Encontro Nacional de Estudantes de Engenharia Metalúrgica, de Materiais e de Minas, 21 a 25 de julho de 2014, São Paulo, SP, Brasil.
} 


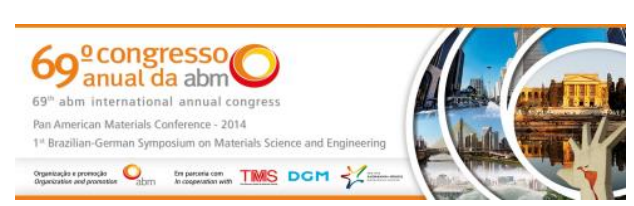

\section{CONCLUSÕES}

Conclui-se que há diferença entre os métodos na determinação do limite de escoamento, do módulo de elasticidade e da deformação final, sendo que a diferença pode chegar a valores da ordem de $37 \%$. No caso do limite de resistência, não é perceptível nenhuma diferença entre os métodos.

Foi realizada uma análise da variância para as propriedades em que houve diferença, e o teste concluiu que essa diferença não é superior ao erro aleatório entre as amostras. Ainda assim, dependendo da aplicação dos resultados, pode ser importante realizar a análise e correção dos dados obtidos no ensaio.

\section{REFERÊNCIAS}

1 Garcia A, Spim JA, Santos CA. Ensaios dos Materiais. 2ª edição. Rio de Janeiro: LTC; 2012.

2 Souza SA. Ensaios Mecânicos de Materiais Metálicos - Fundamentos Teóricos e Práticos. 5a ed. São Paulo: Ed. Edgard Blücher; 1982.

3 Dieter G. Metalurgia Mecânica. Rio de Janeiro: Guanabara Dois; 1981.

4 Meyers MA, Chawla KK. Mechanical Behavior of Materials. 2008.

5 Ribeiro Jld, Caten CST. Série Monográfica Qualidade: Projeto de Experimentos. Porto Alegre: UFRGS; 2000.

6 American Society for Testing and Materials. ASTM E8/E8M: Standard Test Methods for Tension Testing of Metallic Materials. West Conchohocken; 2013.

7 International Organization for Standardization. ISO 6892-1: Tensile Testing of Metallic Materials - Part 1: Method of Test at Room Temperature. Geneva; 2009.

\footnotetext{
* Contribuição técnica ao $69^{\circ}$ Congresso Anual da ABM - Internacional e ao 14ํㅡㄹ ENEMET - Encontro Nacional de Estudantes de Engenharia Metalúrgica, de Materiais e de Minas, 21 a 25 de julho de 2014, São Paulo, SP, Brasil.
} 